\title{
Particle Physics Models of Inflation in Supergravity
}

\author{
Stefan Antusch*, Philipp M. Kostka and Koushik Dutta \\ Max-Planck-Institut für Physik (Werner-Heisenberg-Institut), \\ Föhringer Ring 6, 80805 München, Germany \\ E-mail: \\ antusch@mppmu •mpg ·de,kostka@mppmu.mpg • de,koushik@mppmu .mpg ·de
}

While there exists a large multitude of inflationary models, the connection of inflation to particle physics is still an unsolved puzzle. In particular, in supergravity theories, where the so-called $\eta$ problem tends to spoil slow-roll inflation, the construction of convincing and technically natural models of inflation is challenging. We discuss some recent developments regarding the quest for particle physics models of inflation in supergravity.

European Physical Society Europhysics Conference on High Energy Physics

July 16-22, 2009

Krakow, Poland

${ }^{*}$ Speaker. 


\section{Introduction}

Inflation model building offers a multitude of possibilities for realizing inflation [1]. Among the many classes of models, hybrid inflation is especially promising to make a connection between the inflationary paradigm and particle physics: The "waterfall" ending hybrid inflation may be associated with particle physics phase transitions such as the spontaneous breaking of the gauge group of a grand unified theory or a flavor symmetry. In SUGRA, which provides a solution to the hierarchy problem associated with such new physics at high energies, the $\eta$-problem is well known to put inflation models under considerable pressure [2]. In this talk, we discuss a new variant of hybrid inflation models within SUGRA, referred to as "tribrid inflation", where in addition to the inflaton and waterfall field, the model contains a third 'driving' field which contributes the large vacuum energy during inflation by its F-term. We discuss how the $\eta$-problem of SUGRA inflation can be solved in "tribrid inflation" using either a Heisenberg symmetry or a shift symmetry of the Kähler potential.

\section{Tribrid Inflation}

In the following, we will discuss inflationary models of the "tribrid inflation" type, where the superpotential is given by

$$
W=\kappa S\left(H^{2}-M^{2}\right)+g(\Phi, H) .
$$

We denote the chiral superfield containing the slow-rolling inflaton as scalar component by $\Phi$ and the one containing the waterfall field by $H$. The F-term of the field $S$ contributes the vacuum energy that drives inflation in this class of models whereas inflation ends when the waterfall field acquires a vacuum expectation value (vev) $\langle H\rangle \sim M$. For simplicity, we use only singlet fields. Using gauge multiplets, one would substitute $H^{2} \rightarrow H \bar{H}$, with $\bar{H}$ being another field in the conjugate representation. Note that in contrast to the "standard" SUSY hybrid inflation models [2, 3], where the driving field is identical with the inflaton, in tribrid inflation each of the three main ingredients of the inflationary model is distributed to a separate field ${ }^{1} . S$ stays at zero during inflation and only contributes the large vacuum energy by its F-term. A large mass stabilizing $\mathrm{S}$ at zero is typically generated by SUGRA effects from generic non-minimal Kähler potentials. $\Phi$ is the flat inflaton direction which slow-rolls and stabilizes $H$ via the coupling $g(\Phi, H)$ until $\Phi$ reaches a critical value and thus triggers the waterfall. This ends inflation due to the fact that $H$ develops a tachyonic mass squared and quickly falls towards the true vacuum $\langle H\rangle \sim M$. With $S=H=0$ during inflation, tribrid inflation satisfies $W=W_{\Phi}=0$ during inflation.

\section{Tribrid Inflation and Solutions to the $\eta$-Problem}

With a general expansion of the Kähler potential in terms of fields over some cutoff scale and a suitable adjustment of the expansion parameters, it is always possible to "tune away" the $\eta$-problem in both the "standard" hybrid [4] and the tribrid inflation scenarios [5].

However, if one attempts to solve the $\eta$-problem by a fundamental symmetry in the Kähler potential, this turns out to be extremely difficult to achieve in "standard" hybrid-type models [6,7].

\footnotetext{
${ }^{1}$ Hence the name tribrid inflation.
} 
The reason for this is that such symmetries typically lead to a tachyonic direction in the potential which can only be stabilized at the cost of some extra complications, for instance by using the couplings to additional moduli fields which themselves have stabilization problems and induce dangerous couplings to the inflaton via the SUGRA F-term scalar potential ${ }^{2}$

$$
V_{F}=e^{K}\left[K^{i \bar{j}} D_{i} W D_{\bar{j}} \bar{W}-3|W|^{2}\right],
$$

where the derivative $D_{i} W \equiv W_{i}+W K_{i}$ has been introduced.

These problems do not arise if one combines the tribrid scenario in Eq. 2.1 with a symmetry protecting the Kähler potential. The main reason is that the vanishing of the inflationary superpotential $W_{\text {inf }}$ and its derivative with respect to the inflaton during inflation avoids the appearance of tachyonic directions in the potential. In addition, various potentially dangerous terms in the scalar potential, concerning for example couplings to moduli fields, are automatically absent in tribrid inflation compared to the "standard" hybrid case. We now discuss two realizations of tribrid inflation in supergravity where the $\eta$-problem is solved naturally by either a Heisenberg symmetry or a shift symmetry of the Kähler potential $[8,9]$.

\subsection{Heisenberg Symmetry Solution}

As a specific realization of tribrid inflation, in Ref. [8] we have considered the superpotential in Eq. 2.1 with (c.f. [5])

$$
g(\Phi, H)=\frac{\lambda}{M_{*}} \Phi^{2} H^{2}
$$

in combination with a Heisenberg symmetry invariant Kähler potential of the form

$$
K=|H|^{2}+\left(1+\kappa_{S}|S|^{2}+\kappa_{\rho} \rho\right)|S|^{2}+f(\rho) .
$$

The invariant combination under the non-compact Heisenberg group transformations is given by $\rho=T+T^{*}-|\Phi|^{2}$.

The Heisenberg symmetry of the Kähler potential, or in other words the fact that $K$ depends on $\rho$ only, together with the absence of kinetic mixing in the $(\rho, \Phi)$-basis, protects the potential Eq. 3.1 from containing SUGRA corrections to the mass of the inflaton $|\Phi|$. We have shown that it is possible to stabilize the modulus $\rho$ by the additional coupling $\kappa_{\rho}$ with the help of the vacuum energy during inflation. While the Heisenberg symmetry solves the $\eta$-problem by keeping the treelevel potential exactly flat in $|\Phi|$-direction, one-loop corrections due to the Heisenberg symmetry breaking operator 3.2 with the waterfall sector fermions, scalars and pseudoscalars running in the loops lift the flatness of the potential and generate the slope necessary for slow-roll inflationary dynamics.

\subsection{Shift Symmetry Solution}

As another realization of the tribrid scenario, in Ref. [9] we have considered the superpotential in Eq. 2.1, again with the same function $g(\Phi, H)$ defined in Eq. 3.2. This has been combined with a Kähler potential

$$
K=|H|^{2}+|S|^{2}+\frac{1}{2}\left(\Phi+\Phi^{*}\right)^{2}+\frac{\kappa_{S}}{\Lambda^{2}}|S|^{4}+\frac{\kappa_{\Phi}}{4 \Lambda^{2}}\left(\Phi+\Phi^{*}\right)^{4}+\frac{\kappa_{S H}}{\Lambda^{2}}|S|^{2}|H|^{2}+\ldots,
$$

\footnotetext{
${ }^{2}$ We use units where we set the reduced Planck scale $M_{P} \sim 2.4 \cdot 10^{18} \mathrm{GeV}$ to one.
} 
where the ellipsis symbolize all possible similar terms of the same order and the suppressed higher order terms. For fairly generic values of the couplings in the Kähler potential, it is possible to make all scalars in the theory except for the inflaton heavier than the Hubble scale during inflation.

Due to the shift symmetry $\Phi \rightarrow \Phi+i \mu$ in the Kähler potential we obtain a tree-level flat inflaton direction $\phi_{I}=\sqrt{2} \operatorname{Im}(\Phi)$ and hence evade the $\eta$-problem. Again, radiative corrections induced by the shift symmetry breaking term in Eq. 3.2 lift the flatness of the potential. For sufficiently large values of the parameter $\kappa_{S H}$, the loop-corrected potential can be of hilltop-form leading to a stronger negative curvature of the inflaton potential and finally allow for a reduced spectral index consistent with best-fit values to the WMAP 5 year data.

\section{Summary and Conclusions}

In summary, we have discussed how the $\eta$-problem of SUGRA inflation can be solved in a novel class of F-term inflation, which we have referred to as tribrid inflation. When tribrid inflation is combined with a Heisenberg or shift symmetry invariant Kähler potential, higher order operators from the SUGRA expansion that give rise to the $\eta$-problem are forbidden. Furthermore, due to the properties of $W=W_{\Phi}=0$ during inflation, tribrid inflation avoids stability problems which appear when "standard" hybrid inflation models are combined with fundamental symmetries in the Kähler potential. Therefore, we conclude that tribrid inflation is tailor-made for solving the $\eta$-problem by symmetries in the Kähler potential. Furthermore, it also allows for attractive connections to particle physics: The right-handed sneutrino, for example, provides an interesting inflaton candidate in tribrid inflation.

\section{Acknowledgments}

The authors would like to thank Steve F. King and Mar Bastero-Gil for collaboration in part of the works presented here. The authors acknowledge partial support by the DFG cluster of excellence "Origin and Structure of the Universe".

\section{References}

[1] For a review, see e.g.: D. H. Lyth and A. Riotto, Phys. Rept. 314, 1 (1999).

[2] E. J. Copeland, A. R. Liddle, D. H. Lyth, E. D. Stewart and D. Wands, Phys. Rev. D 49, 6410 (1994).

[3] G. R. Dvali, Q. Shafi and R. K. Schaefer, Phys. Rev. Lett. 73, 1886 (1994).

[4] M. Bastero-Gil, S. F. King and Q. Shafi, Phys. Lett. B 651, 345 (2007).

[5] S. Antusch, M. Bastero-Gil, S. F. King and Q. Shafi, Phys. Rev. D 71 (2005) 083519.

[6] P. Brax, C. van de Bruck, A. C. Davis and S. C. Davis, JCAP 0609, 012 (2006).

[7] S. C. Davis and M. Postma, JCAP 0804, 022 (2008).

[8] S. Antusch, M. Bastero-Gil, K. Dutta, S. F. King and P. M. Kostka, JCAP 0901, 040 (2009).

[9] S. Antusch, K. Dutta and P. M. Kostka, Phys. Lett. B 677, 221 (2009). 